\title{
-NOTE-
}

\section{A MIXED BOUNDARY-VALUE PROBLEM FOR FIBER-REINFORCED MATERIALS*}

By A. C. PIPKIN and T. G. ROGERS

(Brown University)

1. Introduction. In a previous paper [1] we have outlined a theory of plane deformations of materials which are both incompressible and reinforced with inextensible fibers. We showed that, under weak restrictions on the shear stress response of the material, every kinematically admissible deformation is also statically admissible. The analysis was not restricted to any particular material behavior such as elasticity, plasticity or viscoelasticity.

In this note we consider a specific mixed boundary-value problem in which more than one kinematically admissible deformation satisfies the prescribed displacement boundary conditions. The purpose of the note is to show how to determine whether or not a given kinematically admissible deformation furnishes a solution, by using the prescribed traction boundary conditions. For this purpose, it is necessary to describe the shear response of the material a little more specifically than would be necessary in a pure displacement boundary-value problem.

2. Statement of the problem. The problem involves a slab of material initially bounded by planes $X_{1}=0, H$ and $X_{2}= \pm L$ (Fig. 1). The slab is so constrained that

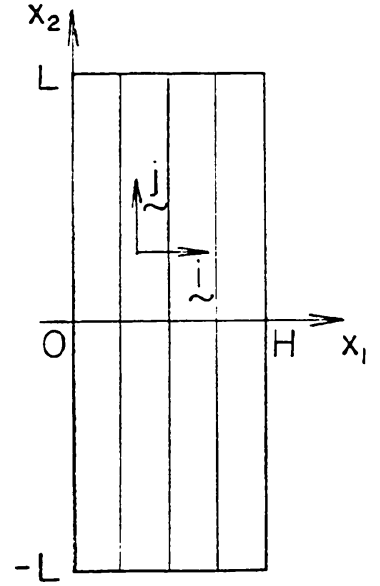

FIG.1

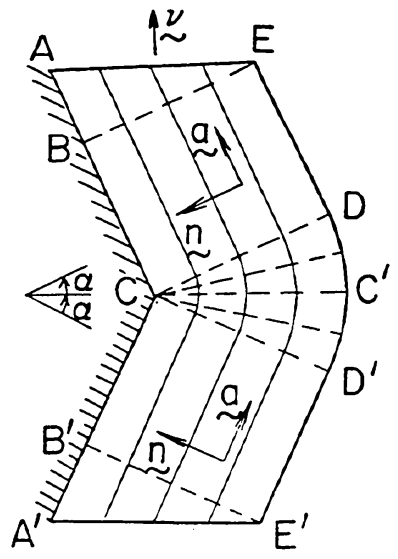

Fig. 2

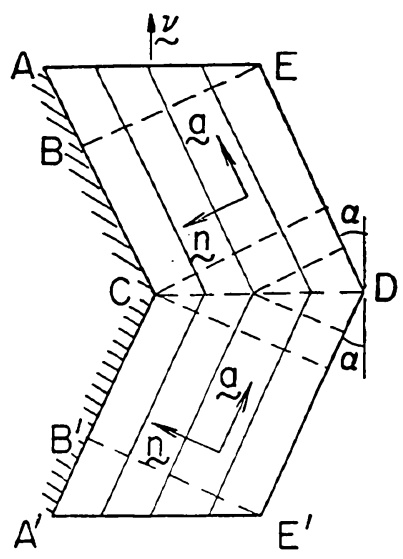

Fra. 3

*Received February 11, 1970. 
we can assume conditions of plane strain and ignore the coordinate $X_{3}$. The final position $\mathbf{X}$ of a particle initially at $\mathbf{X}$ is then given by

$$
x_{1}=x_{1}\left(X_{1}, X_{2}\right), \quad x_{2}=x_{2}\left(X_{1}, X_{2}\right), \quad x_{3}=X_{3} .
$$

The slab is composed of homogeneous, incompressible material, and it is reinforced with inextensible fibers that are initially straight and parallel to the $X_{2}$ direction. The fibers are treated as being continuously distributed. Thus any material curve that initially lies along a line $X_{1}=$ constant is called a fiber, and no segment of any such curve can change length in any kinematically admissible deformation.

The slab is deformed so that its side $X_{1}=0$ lies along a rigid wall $A B C B^{\prime} A^{\prime}$ with two straight segments $A B C$ and $C B^{\prime} A^{\prime}$ which intersect at an angle $2 \alpha$ (Figs. 2 and 3). The particle initially at $X_{1}=X_{2}=0$ lies at the corner $C$ and the remaining particles on the fiber $X_{1}=0$ take positions determined by the inextensibility condition. The slab is bonded to the wall, and the remaining surfaces are then left free of traction.

3. Kinematics. Let a be a field of unit vectors tangential to the fibers in the deformed state, and let $\mathbf{n}$ be a field of unit vectors orthogonal to a at each point (Figs. 2 and 3). The curves to which $\mathbf{n}$ is tangent are called normal lines.

The constraints of incompressibility and inextensibility imply [1] that normal lines must be straight when the fibers are initially parallel. Their orthogonal trajectories, the fibers, are then parallel curves and the constraints imply [1] that the distance between any two fibers must be the same as it was initially.

Figs. 2 and 3 show two deformations that satisfy the constraint conditions and the specified displacement boundary condition on the fiber $X_{1}=0$, for tan $\alpha$ less than $L / H$. The deformation shown in Fig. 2 is proposed because it seems to be the most obvious deformation we should expect. If we think of the corner at $C$ as slightly rounded off, then the straight normal lines perpendicular to the fiber $A B C B^{\prime} A^{\prime}$ must be as shown in Fig. 2. Nevertheless, Fig. 3 is also kinematically admissible, and among deformations that involve discontinuities of the fiber directions, it seems to be the simplest possibility. Both of these deformations, and all others that are kinematically admissible, can be supported in equilibrium with suitable tractions on the boundary [1]. However, as we shall see, the prescribed conditions of zero traction are satisfied by the deformation in Fig. 2 but not, in general, by that in Fig. 3, provided that the shearing stress response of the material satisfies some weak and natural conditions.

4. Amount of shear. In evaluating the shearing stress it is necessary to know the local state of deformation at each particle. This information is obtained by noting that a material line element $\mathbf{j} d X_{2}$ deforms into an element a $d X_{2}$, and an element $\mathbf{i} d X_{1}$ becomes $-(\mathbf{n}+\gamma \mathbf{a}) d X_{1}$. The coefficient $\gamma$ defines the amount of shear at the particle considered. Thus

$$
\partial \mathrm{x} / \partial X_{1}=-\mathrm{n}-\gamma \mathrm{a}, \quad \partial \mathrm{x} / \partial X_{2}=\mathrm{a} .
$$

In Fig. 2, within the fan $C D^{\prime} C^{\prime} D$, the final polar coordinates $r, \theta$ of a particle initially at $X_{1}, X_{2}$ are

Then

$$
r=X_{1}, \quad \theta=X_{2} / X_{1}
$$

$$
\frac{\partial \mathbf{X}}{\partial X_{1}}=\frac{\partial}{\partial X_{1}}\left[r \mathbf{i}_{r}(\theta)\right]=\frac{\partial r}{\partial X_{1}} \mathbf{i}_{r}(\theta)+r \mathbf{i}_{r}^{\prime}(\theta) \frac{\partial \theta}{\partial X_{1}}=\mathbf{i}_{r}-\theta \mathbf{i}_{\theta}
$$


Here $i_{r}$ and $i_{\theta}$ are the unit vectors for the polar system, equal to $-\mathbf{n}$ and a respectively. Then comparison with (4.1) shows that the amount of shear is

$$
\gamma=\theta \text {. }
$$

In the region $A C D E, \gamma$ is everywhere equal to its value $\alpha$ on $C D$, and in $A^{\prime} C D^{\prime} E^{\prime}, \gamma$ is equal to $-\alpha$.

In Fig. 3 the values of $\gamma$ in the upper and lower halves are respectively $\tan \alpha$ and $-\tan \alpha$.

5. Stress and equilibrium. Assuming that the stress components $\sigma_{31}$ and $\sigma_{32}$ are zero by symmetry, the stress for a plane deformation is of the form [1]

$$
\mathbf{o}=-p(\mathbf{I}-\mathrm{aa})+T \mathrm{aa}+S_{33} \mathrm{k} \mathrm{k}+S(\mathrm{an}+\mathrm{na}) .
$$

Here we use dyadic notation; for example, $T a$ a is the tensor with cartesian components $T a_{i} a_{i} . I$ is the unit tensor and $\mathbf{k}$ is a unit vector in the $X_{3}$ direction. $T$ and $p$ are reactions to the constraint conditions, to be determined from the equilibrium equations. $S$ and $S_{33}$ are functions of the amount of shear or its history, with forms which depend on specific material properties such as elasticity, plasticity or viscoelasticity.

Assuming that $\partial S_{33} / \partial X_{3}$ is zero in plane deformations, the equilibrium equations with zero body force yield [1]

$$
\begin{gathered}
\mathbf{a} \cdot \nabla T+\mathbf{n} \cdot \nabla S-2 \kappa S=0, \\
-\mathbf{n} \cdot \nabla p+\kappa(p+T)+\mathbf{a} \cdot \nabla S=0,
\end{gathered}
$$

and $\partial p / \partial X_{3}=0$. Here $\kappa$ is the curvature of the fiber at the point considered.

A vector v normal to the surface $A E$ is given by

$$
\mathbf{v}=\mathbf{a}-\boldsymbol{\gamma} \mathbf{n} .
$$

The condition of zero traction on $A E(\boldsymbol{\delta} v=0)$ then yields, with (5.1),

$$
p=-S / \gamma, \quad T=\gamma S \text { on } X_{2}=L .
$$

Since $\mathbf{n}$ is normal to the outer boundary $X_{1}=H$, the condition of zero traction on that boundary ( $C^{\prime} D E$ in Fig. 2, $D E$ in Fig. 3) gives

$$
p=0, \quad S=0 \quad \text { on } \quad X_{1}=H .
$$

The conditions on the symmetrically disposed boundaries of the lower region are the same.

The stress cannot be evaluated completely until $S$ is specified. For an elastic material, $S$ is a function $S(\gamma)$ of the amount of shear. For plastic materials, if the deformation is carried out with the magnitude of $\gamma$ increasing monotonically, $S$ is again a function $S(\gamma)$ of the amount of shear; the plastic behavior can be nonhardening (perfectly plastic) or work-hardening. In all cases, we suppose that there is enough material symmetry that $S$ is an odd function of $\gamma$ :

$$
S(-\gamma)=-S(\gamma)
$$

This symmetry is necessary if the deformation is to be symmetrical, as shown in Figs. 2 or 3. The functions $p$ and $T$ are then symmetrical about $X_{2}=0$, and it is sufficient to evaluate them in the upper region $X_{2} \geq 0$. 
6. Singular fiber. Within the theory, a single fiber or normal line (which are actually sheets, of course, taking $x_{3}$ into account) can carry a finite load by itself, and must do so if it is a line of shearing stress discontinuity. In the present problem the boundary condition (5.6b) imposes such a discontinuity. The interior values of $S$ at the boundary fiber, $X_{1}=H$, are specified by a function $S(\gamma)$ that can be expected to be zero only when $\gamma$ is zero, in general, whereas the zero traction condition requires $S$ to be zero on the exterior side of the bounding fiber.

The jump discontinuity in $S$ contributes a term $S(\gamma) \delta(r-H)$ to its derivative $\mathbf{n} \cdot \nabla S$ in (5.2). Here $\delta(r-H)$ is the Dirac delta and $r$ is a coordinate constant along fibers, equal to the polar radius $r$ in the fan region of Fig. 2. From (5.2) we observe that the tension $T$ then also has a Dirac delta in the bounding fiber, corresponding to a finite load in that single fiber.

The fiber next to the wall, $X_{1}=0$, may also exhibit such singular behavior, but the situation there is ambiguous until the value of $S$ is prescribed at the wall. Indeed, we are free to prescribe any distribution for $S$; the tension in the bounding fiber will automatically adjust to equilibrate the discontinuity.

7. Solution. We now show that an equilibrium stress field satisfying the prescribed traction boundary conditions (5.5) and (5.6) can be constructed if the deformation is that shown in Fig. 2, but not if the deformation is that shown in Fig. 3.

We first consider Fig. 2. In the homogeneously deformed region $A C D E$, the fiber curvature $\kappa$ is zero. The amount of shear $\gamma$ is constant, so the shearing stress $S$ is also constant except at its jump discontinuity across the outer bounding fiber. Thus, in this region, (5.2) and (5.3) reduce to

$$
\mathbf{a} \cdot \nabla T=-S(\gamma) \delta(r-H) \text { and } \mathbf{n} \cdot \nabla p=0 .
$$

In the fan region $C D^{\prime} C^{\prime} D$ we introduce polar coordinates $r, \theta$. The fiber curvature is then $1 / r$, and the amount of shear is equal to $\theta$, according to (4.4). It follows that the derivative of $S$ along normal lines $\theta=$ constant is zero except at the jump discontinuity across the outer fiber. Hence, in the fan region the equilibrium conditions (5.2) and (5.3) can be written as

$$
(1 / r)(\partial T / \partial \theta)+S(\theta) \delta(r-H)-(2 / r) S(\theta)=0
$$

and

$$
(\partial p / \partial r)+(p+T) / r+(1 / r) S^{\prime}(\theta)=0 .
$$

With the boundary conditions (5.5) and (5.6), in which $\gamma=\alpha$, from (7.1) we obtain

$$
T=\alpha S(\alpha)+(L-s) S(\alpha) \delta(r-H) \text { in } A C D E
$$

and

$$
p=-S(\alpha) / \alpha \text { in } A B E, \quad p=0 \text { in } B C D E .
$$

In (7.4), $s$ denotes arc length along $C^{\prime} D E$, with $s=0$ at the point $C^{\prime}$. From (7.2), with $T$ given at $\theta=\alpha$ by (7.4), we obtain

$$
\begin{aligned}
T=\alpha S(\alpha) & +(L-\alpha H) S(\alpha) \delta(r-H) \\
& +[H \delta(r-H)-2] \int_{\theta}^{\alpha} S\left(\theta^{\prime}\right) d \theta^{\prime} \text { in } C D C^{\prime} D^{\prime}
\end{aligned}
$$


From (7.3), with $p=0$ at $r=H+$, we obtain

$$
\begin{aligned}
r p(r, \theta)= & (H-r)\left[S^{\prime}(\theta)+\alpha S(\alpha)\right]+(L-\alpha H) S(\alpha) U(H-r) \\
& +[H U(H-r)-2(H-r)] \int_{\theta}^{\alpha} S\left(\theta^{\prime}\right) d \theta^{\prime} \text { in } C D C^{\prime} D^{\prime} .
\end{aligned}
$$

Here $U(H-r)$ is the unit step function, equal to unity inside the body but passing discontinuously to zero across the singular boundary fiber.

We now consider the deformation in Fig. 3. If we proceed as in the previous case, we find that $T$ and $p$ must be given by expressions of the forms (7.4) and (7.5), but with $\alpha$ replaced by $\tan \alpha$ everywhere. Now, however, there is a line of discontinuity $C D$ which is neither a fiber nor a normal line. Equilibrium requires that the stress vector $\boldsymbol{d} j$ be continuous across $C D$. But $T, p$ and $S$, and hence $\delta$, have already been determined on both sides of $C D$, and an easy computation shows that the two values of $\delta j$ are not equal, except in the trivial case $S=0$. Thus for Fig. 3 there is no equilibrium stress field compatible with the boundary conditions.

We should immediately point out that for plastic materials, the conclusion that Fig. 3 does not give a solution depends strongly on the assumption that there has been no unloading. For example, if a body of rigid-plastic material were brought into the shape shown in Fig. 3 by applying suitable tractions, and the loads were then removed, the body would retain its shape and be in equilibrium under zero stress.

Acknowledgment. The work described in this note was carried out under grants from the U. S. National Science Foundation and the Advanced Research Projects Agency, NSF Grant GP-9496. We gratefully acknowledge their support.

\section{REFERENCE}

[1] A. C. Pipkin and T. G. Rogers, Plane deformations of incompressible fiber-reinforced materials, J. Appl. Mech. (forthcoming) 